\title{
Acute on Chronic Limb Ischemia Treatment: Case Series
}

\author{
Renaldi Prasetio ${ }^{1 *(\mathbb{D})}$, I Komang Adhi Parama Harta ${ }^{2}$ \\ ${ }^{1}$ Department of Surgery, Faculty of Medicine, Universitas Udayana, Bali, Indonesia; ${ }^{2}$ Department of Surgery, Faculty of Medicine, \\ Division of Cardio Thoracic and Vascular Surgery, Universitas Udayana, Sanglah General Hospital, Denpasar, Bali, Indonesia
}

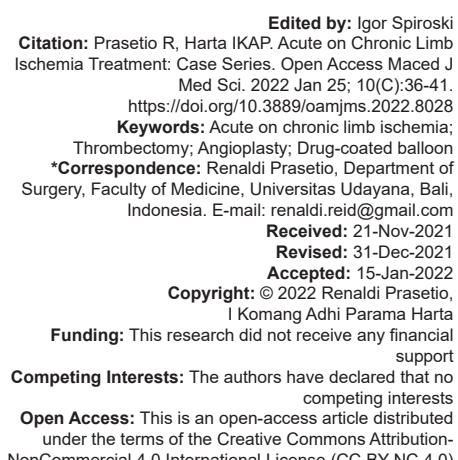

\section{Abstract}

BACKGROUND: Acute on chronic limb ischemia is a condition caused by acute disruption in chronic vessel disease The major cause is peripheral arterial disease or embolism. Treating the acute phase while considering the origin of disease is important to treat acute on chronic limb ischemia.

CASE DESCRIPTION: Three cases were described on this serial case report. The first and second cases represent acute on chronic limb ischemia conditions with a history of intermittent claudication (IC) and signs of acute limb ischemia (ALI) upon arrival. They were both treated with surgical intervention for ALI. Despite of adequate surgica intervention, both cases showed suboptimal limb recovery. Both patients were referred for further evaluation of chronic vessel disease with angiography. Third case represents chronic limb ischemia as endpoint of treatment from the first and second cases. Patient with worsening IC despite adequate medical and exercise treatment underwent angiography examination. Angiography results found two sites of total occlusion of the right common iliac artery and superficial femoral artery (SFA). Angioplasty and primary stenting were done on the common iliac artery. Drug coated balloon and stenting were also performed on SFA.

CONCLUSION: Patients with acute on chronic limb ischemia need to be treated during acute phase and the remaining chronic vessel disease. Consideration and care should be taken when managing similar cases for optimal treatment.

\section{Introduction}

Limb ischemia refers to the symptomatic disruption of arterial blood flow to the extremities and is classified into acute and chronic limb ischemia. Peripheral arterial disease (PAD) is the leading cause of both conditions [1]. PAD affects over 200 million individuals worldwide. Chronic limb ischemia is characterized by the formation of flow-limiting stenotic and occlusive atherosclerotic plaques with symptoms more than 2 weeks. The condition is uncommon in the under 50 age group but prevalence rises to approximately $20 \%$ in the over $80 \mathrm{~s}$ [2]. In contrast, acute limb ischemia (ALI) is defined as a sudden disruption of limb perfusion that endangered the viability of the limb [3]. The disease is characterized by either thrombotic or embolic occlusion of a peripheral artery and symptoms are of $<2$ weeks duration. In patients with PAD, "acute-onchronic" ischemia represents an acute deterioration in their symptoms [4]. Here we present patients with acute on chronic limb ischemia. The first two cases were with intermittent claudication (IC) and signs of ALI. Third case was patient with chronic limb ischemia which act as the end evaluation and treatment from the previous case.

\section{Case Presentation}

\section{Case 1}

A 61-years old male presented with pain on his left toes which started around 8-9 months prior. $\mathrm{He}$ had a consultation with vascular surgeon and was prescribed clopidogrel $75 \mathrm{mg}$ once daily for 8 months. Initially, the symptoms improved, however, it started to get worse since yesterday. He had pain during resting and worsened during walking and movement. $\mathrm{He}$ also noticed his left toes become more blueish and felt minimal sensation on it. He was still able to walk and move his left leg with pain. There was no history of trauma on the affected leg. No allergic history. Past medical history was asthma. He smoked cigarette for 45 years and stopped only 3 days prior.

Initial physical examination revealed stable vital sign. Local state on left lower leg showed cyanotic on all toes until base of the metatarsal area, scar on the base of great toe and point toe without any bleeding nor swelling. The skin was cold with minimal sensibility on all toes and capillary refill time of $<2 \mathrm{~s}$. Pulse on dorsalis pedis artery was not palpable. Pulse oximetry on all toes was undetected with normal range of motion on toes and ankle. Patient was at Ila clinical-stage from Rutherford. 
Computed tomography angiography (CTA) demonstrated abrupt thrombotic narrowing and cutoff at the left distal superficial femoral artery (SFA) (Figure 1). Intravenous Heparin $100 \mathrm{IU} \mathrm{kg/body} \mathrm{weight}$ was initiated continued with maintenance of $10 \mathrm{lU} /$ $\mathrm{kg}$ with target activated partial thromboplastin time (aPTT) of 1.5-2.5 times normal value. Painkiller was administered.

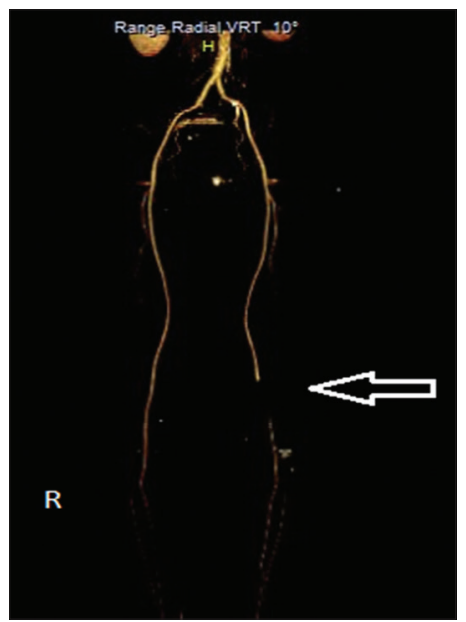

Figure 1: Digital reconstruction of computed tomography angiography of lower extremities showing abrupt thrombotic narrowing and cut-off at the left distal superficial femoral artery (SFA) (left distal SFA) with minimal collaterals blood vessels

Surgical thrombectomy with Fogarty catheter was done but unable to reach the distal part of the extremity. Thrombus was evacuated with the proximal and distal flow were present during evaluation. IV Heparin for maintenance was continued. During the hospital course patient's condition improved, pain subsided, able to mobilize well without pain, and blueish on all toes subsided. However, oxygen saturation on all toes ranged around $90-95 \%$ indicating suboptimal recovery. Patient was discharged on day 5 and planned to have angiography on the lower extremity for further evaluation.

\section{Case 2}

A 59-year-old male presented with worsening pain on the right lower leg 1 week before hospital admission. He had a history of on and off pain on his right lower leg since 4 years ago. He had consultation with a Cardiologist and was given Aspirin $100 \mathrm{mg}$ once daily and Clopidogrel $75 \mathrm{mg}$ once daily which also related to his heart diseases. Since yesterday, he was unable to walk more than 5 min without excruciating pain. Denied any history of trauma or allergies. Past medical history included congestive heart failure due to coronary artery disease with history of the coronary arterial bypass graft, type II diabetes mellitus, chronic obstructive pulmonary disease, and dyslipidemia, all in regular medications. Patient smoked around 40 years and stopped 2 years ago.

Initial physical examination revealed stable vital sign. Local state of the right lower leg showed cyanotic toes with tenderness and cold extremity. Sensibilities were decreased on all toes until dorsalis pedis, CRT $<2 \mathrm{~s}$, pulse oximetry on all toes were decreased ranging from $85 \%$ to $96 \%$ with decreased ROM on toes and ankle. Pulse on the right dorsalis pedis was not palpable. Patient was classified into the clinical stage of Rutherford Ilb. Laboratory examinations showed increased $\mathrm{HbA} 1 \mathrm{c}$ (7.6 \%) and other parameter within normal range. Patient underwent CTA which demonstrated wall plaque calcifications along the right SFA with widening and intraluminal thrombosed within proximal part of the right SFA just below the bifurcation causing abrupt occlusion. Wall plaque calcifications along left SFA also seen with partial intraluminal thrombosed (Figure 2).

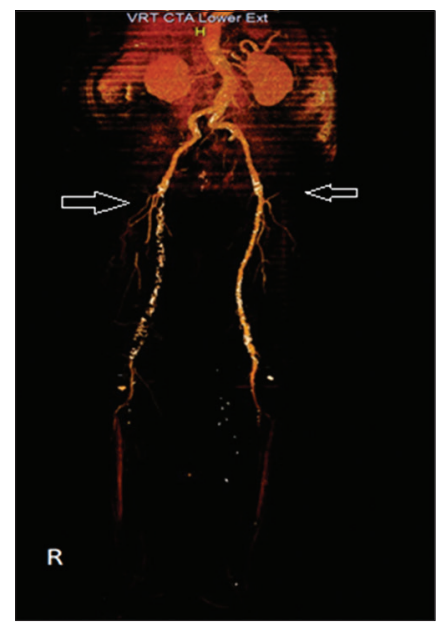

Figure 2: Computed tomography angiography of lower extremity showing Wall plaque calcifications along right SFA with widening and intraluminal thrombosed within proximal part of right SFA just below the bifurcation causing abrupt occlusion with minimal collateral blood vessels

Intravenous Heparin $100 \mathrm{IU}$ kg/body weight was initiated and $10 \mathrm{lU} / \mathrm{kg}$ maintenance with target aPTT of 1.5-2.5 times normal value. Intravenous painkiller was administered and aspirin and clopidogrel were continued. Surgical thrombectomy with Fogarty catheter was done until distal part of extremity, thrombus was evacuated. The proximal and distal flow were present during the evaluation. IV Heparin drip maintenance. During admission, patient had episode of atrial fibrillation with rapid ventricular response. He was consulted to cardiologist and treated accordingly. Echocardiography showed normal cardiac output without any thrombi on the cardiac chamber.

After 9 days of admission, patient condition generally improved with alleviation of pain and able to mobilize. However, oxygen saturation on all toes ranged around $92-95 \%$ which resulted in suboptimal recovery. Patient then planned to have angiography on the lower extremity for further evaluation.

\section{Case 3}

A 51-years old male presented with pain on right leg one year before hospital admission. 
Pain occurred during walking around $100 \mathrm{~m}$ and subsided with rest. He has a history of type II diabetes mellitus, hypertension, and dyslipidemia all on regular medications. Two months ago, he started to notice his foot became colder than usual but did not see any bluish colors on his toes. He had consultation with vascular surgeon and was prescribed Aspirin $85 \mathrm{mg}$ once daily and Cilostazole $100 \mathrm{mg}$ twice daily. Combined with exercise therapy, his symptoms were improved. Patient denied any significant family history and history of trauma. Patient smoked for 28 years and stopped 1 year ago.

Initial physical examination revealed stable vital signs. Examination on both lower extremities showed palpable dorsalis pedis pulse, normal sensibility and CRT of $<2 \mathrm{~s}$. Both extremities have normal ROM. Other physical examinations were not significant. He was classified into clinical-stage Rutherford 3 and Fontaine $2 \mathrm{~b}$.

Patient underwent CTA of lower extremities which showed: Significant atherosclerotic changes with multiple calcific plaques with significant luminal narrowing in the right external Iliac artery, right internal iliac artery right SFA, and left SFA with collateral blood vessels (Figure 3 ).

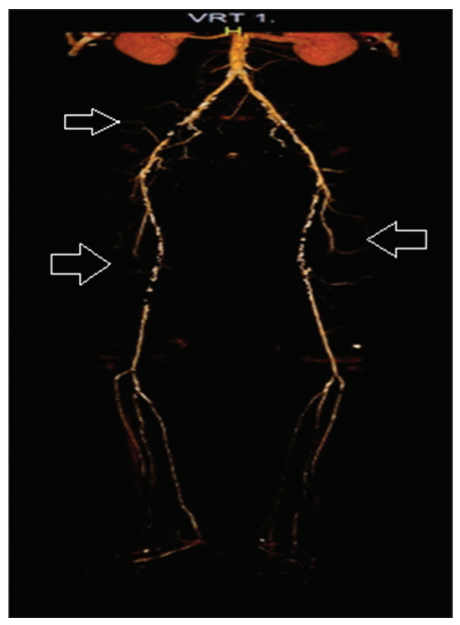

Figure 3: Computed tomography angiography on lower extremities showing Significant atherosclerotic changes with multiple calcific plaques with significant luminal narrowing in right External Iliac artery, right Internal Iliac artery, right superficial femoral artery (SFA), and left SFA with collateral blood vessels

Angiography on right peripheral extremities showed total occlusion in the right common iliac artery and right SFA $(<15 \mathrm{~cm})$ with collaterals artery found (Figures $4 a$ and $5 a$ ). Patient was categorized as TransAtlantic Inter-Society Consensus Classification (TASC) B lesions.

Following the angiography result, balloon angioplasty and stenting were conducted on the right CIA (Figure 4b). Drug coated balloon (DCB) angioplasty and stent on right SFA (Figure 5b). Arteriography post stent showed favorable artery blood flow On CIA and SFA (Figures $4 c$ and $5 c$ ). Patient was discharged with significant improvement. Pain subsided and able to mobilize well.

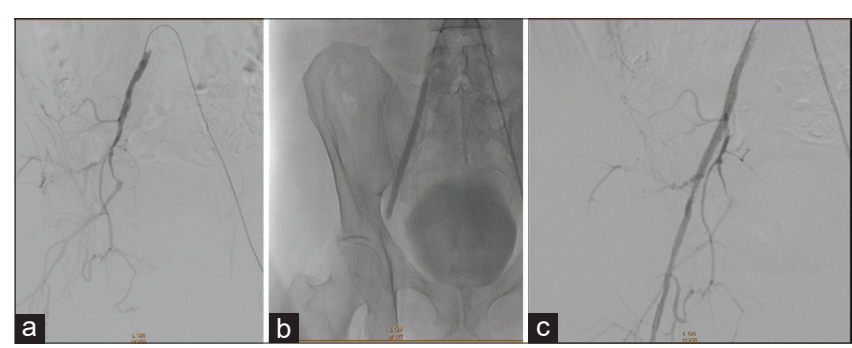

Figure 4: (a) Total occlusion on right common iliac artery (CIA). (b) Punctured on left femoral artery, cross to the right femoral artery. JR 4.0 $5 \mathrm{Fr}$ catheter to proximal right iliac artery. Wire passed the occlusion. Dilatation with balloon $4 \mathrm{~mm}$ and $7 \mathrm{~mm}$. Stent Innova $7 x$ $150 \mathrm{~mm}$ was placed (c) Favourable blood flow on CIA after balloon and stent placement

\section{Discussion}

From the first and second cases, patient presented with signs of ALI of 6P (pain, pallor, pulseless, perishing cold, paraesthesia, paralysis). They also had a history of IC. Since both patients suffered from CLI, the acute event could be trigger by thrombotic occlusions or embolic occlusion on stenosis blood vessels representing acute on chronic ischemia. Thrombotic occlusions can be due to the rupture of an atherosclerotic plaque or in situ thrombosis. While embolism in ALI can be caused by cardiogenic or cholesterol emboli. On cardiogenic emboli, it can be due to atrial fibrillation and embolization of a mural thrombus. Cholesterol emboli can occur in rupture of proximal atherosclerotic plaque or embolus from the proximal aneurysm [4], [5].

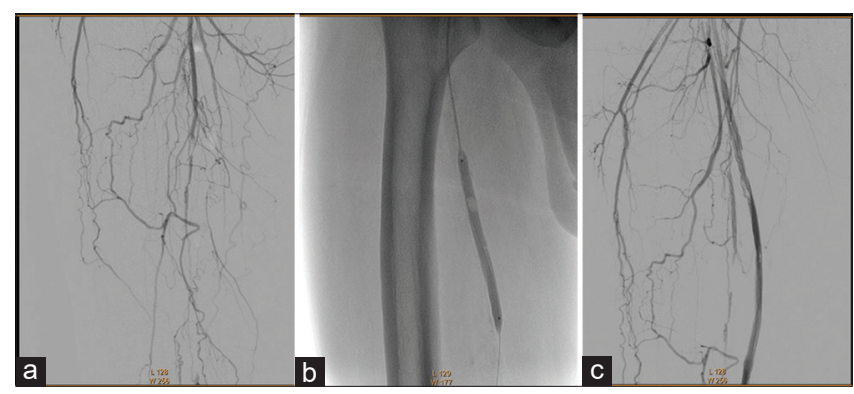

Figure 5: (a) Total occlusion on right superficial femoral artery (SFA). (b) Catheter inserted to the proximal right SFA with collaterals found. Dilatation with balloon $7 \mathrm{~mm}$ and drug coated balloon (DCB) $6 \mathrm{~mm}$. First Supera Peripheral Stent $5.5 \mathrm{~mm}$ was placed at distal femoral artery, continued with second Supera Peripheral Stent 6.5 placed proximal to the first stent graft (c) Favourable blood flow on SFA after DCB and stent placement

Clinical classification by Rutherford can be used to determine the severity of ischemia. First case was classified as Rutherford Ila because of normal motor function with decreased of sensibility of toes. The second case patient was classified as Rutherford IIb because of sensory loss until dorsalis pedis and mild to moderate muscle weakness [4]. Patient also had a history of AF during hospitalization, but previous AF events were unknown.

Imaging study prior to treatment is important. CTA is usually the preferred choice due to its availability 
and non-invasive. Duplex can be effective but rather operator-dependent with bowel gas obscuring the related abdominal vessels. DSA is still the gold standard for evaluation and treatment but is not often used prior to previous imaging [4]. Based on CTA examination, both cases showed acute on chronic limb ischemia due to total occlusion. Second case also showed chronic vessel disease on the contralateral part.

In ALI, systemic anticoagulation with unfractionated heparin needs to be immediately given to decrease the risk of further clot propagation and to prevent microvascular thrombosis. An initial bolus of $100 \mathrm{Units} / \mathrm{kg}$ is given, followed by an intravenous infusion of 1000 Units/h. If urgent revascularization is not undertaken, the heparin dose should be titrated to maintain APTT between 50 and $80 \mathrm{~s}$ or 2.0-3.0 times normal values. For patients with heparin allergy, direct thrombin inhibitor can be used and titrated to an aPTT of 50-80 s [6].

Revascularization in $\mathrm{ALI}$ can range from surgical thrombectomy, bypass surgery, endovascular treatment (EVT), and hybrid treatment. Thrombectomy using Fogarty catheter has significantly simplified surgical treatment which allows exposure in more convenient location. EVT can range from catheterdirected thrombolysis until percutaneous mechanical thrombectomy. Available facilities and clinical expertise should be considered when deciding the revascularization strategy [7]. Rutherford Class I in ALI can be treated with medical therapy or revascularization electively. Class Ila can be treated with endovascular (more preferred) or surgical treatment (if ischemic symptoms occur more than 2 weeks). Class IIb is preferred with surgical revascularization. Class III is not indicated for immediate revascularization instead requires amputation [8].

On the first and second cases, surgical thrombectomy with Fogarty was done which showed adequate result for treating acute phase. Despite proximal and distal blood flow were present and palpable distal pulse, patient's distal oxygen saturation still showed sub-optimal recovery ranging from $90 \%$ to $95 \%$. Thus, both patients were referred for further angiography after discharged for further evaluation of underlying stenosis or occlusive lesions. Depending on the angiography result, EVT or bypass procedure might be needed. If not treated, this could lead to CLTI in the future. Nomura et al., also found similar case of PAD with CLTI subsequent to ALI. Occlusion on both popliteal arteries was treated with surgical thrombectomy using Fogarty catheter. One month after ALI onset, necrosis of the first toe occurred, which corresponded to the grade of Rutherford class $\mathrm{V}$ for CLTI. Balloon angioplasty was then conducted which showed satisfactory blood flow and wound healing was achieved after 3 months [9].

Third case represents the end-point of evaluation and treatment for the first and second cases. PAD can be caused by chronic atherosclerotic process that progressively narrows the arterial lumen. Longstanding atherosclerosis can form collateral arteries that will help flow to distal extremities. Patient usually presents with IC without any sign of resting pain or ulceration at the lower limb which has already occurs more than 2 weeks [10]. The severity of IC symptoms can be scored by either Rutherford- Baker or Fontaine classification [11]. On the third case, the patient was classified as Rutherford 3 and Fontaine $2 \mathrm{~b}$ as chronic limb ischemia with PAD which differs from CLTI (Rutherford 4-6 and Fontaine 3-4).

Following diagnosis, duplex ultrasound, CTA, MRA still remain the study prior to revascularization. DSA will remain the gold standard for diagnosis and intraoperative evaluation [4]. In our third case, CTA was the faster and available imaging to provide imaging prior to revascularization.

Despite being given exercise and medical therapy patient claudication still persisted. From one compendium of PAD, revascularization is typically based on patient with lifestyle-limiting claudication which no longer responsive to conservative therapy [11]. Based on AHA 2016, revascularization is important for patient functional status and in consequence to quality of life. Surgical and endovascular procedures are effective with patient on lifestyle limiting claudication [7]. TASC II has classified anatomic patterns of disease involvement (types A through D) for aortoiliac and femoropopliteal segment, according to recommended therapy (endovascular versus open surgery) in PAD. Endovascular therapy is the recommended treatment for type A lesions and surgery is the recommended treatment for type D lesions; for TASC B and C lesions, EVT is the preferred treatment for type $B$ lesions and surgery is the preferred treatment for good-risk patients with type $\mathrm{C}$ lesions [3]. On the third case, angiography showed right common iliac occlusion and SFA occlusion $(<15 \mathrm{~cm}$ ) with many collateral blood vessel which supplies the distal arteries. Both lesions were categorized as TASC B lesion and preferable treatment choice was EVT.

We decided to performed angioplasty and stenting on CIA and DCB with stenting on SFA in our third case. Over the years, there has been much improvement on endovascular therapy. Initial endovascular approach is recommended in iliac and femoropopliteal diseases [3]. On aortoiliac diseases, balloon angioplasty has shown excellent long-term patency but increased recoil also found on ostial iliac disease and risk of dissection occurred in more complex diseases (total occlusions, ulcerated or calcified lesions, and aneurysmal segments), in consequence increasing the use of primary stenting [11]. A meta-analysis including 958 total patients with more complex aortoiliac disease showed superior patency rates associated with primary stenting [12]. PTA and stenting are preferred treatment modality for aortoiliac disease and has corresponded with 
satisfactory long-term results. Iliac artery occlusions are indicated for primary stenting whereas iliac artery stenoses are preferred for selective stenting [13]. A meta-analysis of femoropopliteal lesion showed that DCB, covered stent (CS), bare-metal stent (BMS) has shown reduction in target lesion revascularization (TLR) compared to balloon angioplasty for over 12 months. DCB, drug-eluting stent, CS, and BMS were associated with significantly improved primary patency at 12 months compared to balloon angioplasty [14]. The Drug-Eluting Balloon in Peripheral Intervention for the-SFA trial randomizing patients on DCB or conventional angioplasty followed by stenting showed promising outcomes of reduced restenosis and TLR at 12 months [15].

\section{Conclusion}

We present case series describing the diagnostic and treatment of patient with acute on chronic limb ischemia, as well as the consideration that need to be taken regarding the disease. Physician should be aware that acute on chronic limb ischemia needs to be managed in the acute phase while also considering the present chronic vessel disease.

\section{Author Contribution}

All of the authors equally contributed to the study from the conceptual framework, data gathering, and data analysis until interpreting the study results.

\section{Ethic Consideration}

All patients in this study have understood and agreed to the use of patient personal data related to the writing of scientific articles. All patients also gave informed consent consciously regarding the use of the data obtained for the preparation of articles to be published in scientific journals.

\section{References}

1. Waton S, Johal A, Heikkila K, Cromwell D. National Vascular Registry Report 2017. London: The Royal College of Surgeons of England; 2017. Available from: https://www.annualreports.
com/HostedData/AnnualReportArchive/W/TSX_WJA_2017. pdf. [Last accessed on 2021 Oct 01].

2. Fowkes FG, Aboyans V, Fowkes FJ, McDermott MM, Sampson UK, Criqui MH. Peripheral artery disease: Epidemiology and global perspectives. Nat Rev Cardiol. 2017;14(3):156-70. http://doi.org/10.1038/nrcardio.2016.179 PMid:27853158

3. Norgren L, Hiatt WR, Dormandy JA, Nehler MR, Harris KA, Fowkes FG. Inter-society consensus for the management of peripheral arterial disease (TASC II). J Vasc Surg. 2007;45(1):567. http://doi.org/10.1016/j.jvs.2006.12.037

PMid:17223489

4. Nickinson A, Bown MJ. Acute and chronic limb ischaemia. Surg (United Kingdom). 2019;37(2):93-101. https://doi.org/10.1016/j. mpsur.2018.12.007

5. O'Connell JB, Quiñones-Baldrich WJ. Proper evaluation and management of acute embolic versus thrombotic limb ischemia. Semin Vasc Surg. 2009;22(1):10-6. http://doi.org/10.1053/j. semvascsurg.2008.12.004

PMid:19298930

6. Kwolek CJ, Fahad S. Acute ischemia: Treatment. In: Sidawy AN, Perler BA, editors. Rutherfor's Vascular Surgery and Endovascular Therapy. $9^{\text {th }}$ ed. Philadelphia, PA: Elsevier; 2019. p. 1326.

7. Gerhard-Herman MD, Gornik HL, Barrett C, Barshes NR, Corriere MA, Drachman DE, et al. 2016 AHA/ACC guideline on the management of patients with lower extremity peripheral artery disease: Executive summary: A report of the American college of cardiology/American heart association task force on clinical practice guidelines. Circulation. 2017;135(12):686-725. http://doi.org/10.1161/CIR.0000000000000501

PMid:28320815

8. Olinic DM, Stanek A, Tătaru DA, Homorodean C, Olinic M Acute limb ischemia: An update on diagnosis and management. J Clin Med. 2019;8(8):1215. http://doi.org/10.3390/jcm8081215 PMid:31416204

9. Nomura T, Tasaka S, Ono K, Sakaue Y, Wada N, Keira N, et al Successful limb salvage by endovascular treatment for critical limb ischemia subsequent to acute limb ischemia. Oxford Med Case Reports. 2019;2019(10):439-42. http://doi.org/10.1093/ omcr/omz103

PMid:31772742

10. Conte MS, Bradbury AW, Kolh P, White JV, Dick F, Fitridge R, et al. Global vascular guidelines on the management of chronic limb-threatening ischemia. J Vasc Surg. 2019;69(6):3S-125. e40. http://doi.org/10.1016/j.jvs.2019.02.016 PMid:31159978

11. Thukkani AK, Kinlay S. Endovascular intervention for peripheral artery disease. Circ Res. 2015;116(9):1599-613. http://doi. org/10.1161/CIRCRESAHA.116.303503 PMid:25908731

12. Saltzman AJ, Stone GW, Claessen BE, Narula A, Leon-Reyes S, Weisz G, et al. Long-term impact of chronic kidney disease in patients with ST-segment elevation myocardial infarction treated with primary percutaneous coronary intervention: The HORIZONS-AMI (harmonizing outcomes with revascularization and stents in acute myocardial infarction) trial. JACC Cardiovasc Interv. 2011;4(9):1011-9. http://doi.org/10.1016/j. jcin.2011.06.012 PMid:21939942

13. Bekken JA, Jongsma $H$, de Vries JP, Fioole B. Self-expanding stents and aortoiliac occlusive disease: A review of the literature. Med Devices Evid Res. 2014;7(1):99-105. http://doi. org/10.2147/MDER.S60594

PMid:24833925 
14. Khan MS, Zou F, Khan AR, Moustafa A, Schmid CH, Baig M, et al Meta-analysis comparing endovascular treatment modalities for femoropopliteal peripheral artery disease. Am J Cardiol. 2020;128:181-8. https://doi.org/10.1016/j.amjcard.2020.05.015 PMid:32650917

15. Liistro F, Grotti S, Porto I, Angioli P, Ricci L, Ducci K, et al.
Drug-eluting balloon in peripheral intervention for the superficial femoral artery: The DEBATE-SFA randomized trial (Drug eluting balloon in peripheral intervention for the superficial femoral artery). JACC Cardiovasc Interv. 2013;6(12):1295-302. http:// doi.org/10.1016/j.jcin.2013.07.010

PMid:24239203 